\title{
Triggered femtoscopy and dense cold matter.
}

\author{
A.Stavinskiy ${ }^{1}$ \\ ITEP \\ 117218, Moscow, B.Cheremushkinskaya 25s, Russia \\ E-mail: stavinskeitep.ru
}

\begin{abstract}
The importance of femtoscopy of triggered (or OFF-LINE selected) rare events is discussed. We propose to study in the laboratory the region of phase diagram corresponding to extremely high baryon density and low temperature. To this end high $\mathrm{p}_{\mathrm{t}}$ central rapidity double cumulative trigger was proposed and tested experimentally at ITEP ion accelerator by FLINT collaboration. FLINT experiment is dedicated to the search and investigation of dense cold matter. Experimental data of the two runs (2007 and 2010) are presented. Photon spectra in $\mathrm{C}+\mathrm{Be}$ interaction at $3.2 \mathrm{GeV} /$ nucleon were measured within the angular interval $35^{\circ}-70^{\circ}$ (1.s.). The measured, photon spectra indicate the dominance of flucton-flucton interaction. Simulation results of the dense cold matter droplets expected in triggered events are presented. Expected space-time characteristics of the droplet and related theoretical problems are discussed.
\end{abstract}

The Seventh Workshop on Particle Correlations and Femtoscopy

University of Tokio, Japan

September $20^{\text {th }}$ to $24^{\text {th }}, 2011$

Speaker 


\section{Introduction}

A new approach to the laboratory study of extremely high baryon density and low temperature phase diagram region is proposed by FLINT collaboration. High $\mathrm{p}_{\mathrm{t}}$ central rapidity double cumulative trigger for this study was tested experimentally at ITEP ion accelerator. Wide experimental program with such a trigger has been discussed in A.Stavinskiy report at WPCF in Kiev[1]. Two main features are important for the proposed approach. To speak about dense cold matter droplet one needs 1) to obtain as many baryons as possible in the interaction volume and 2) to minimize this volume. The higher is $\mathrm{p}_{\mathrm{t}}$ for trigger photon, the large is minimum number of nucleons involved into flucton-flucton interaction(following by D.I.Blokhinzev we'll use the term flucton for a few nucleon short range correlation inside nucleus). Here we present a new experimental data for photon spectra where measured in $\mathrm{C}+\mathrm{Be}$ interaction at $3.2 \mathrm{GeV} /$ nucleon within the angular interval $35^{\circ}-70^{\circ}$ (1.s.).

To estimate the interaction volume of the baryonic system created in the trigger selected events one has to determine the location of this system in phase space. We present here the results of simulations which show the phase space location of the baryonic system under study and the background estimate. Once identified, this baryonic system should be studied by femtoscopic (or alternative) method to estimate the size of the interaction volume. It is a nontrivial task for femtoscopy both because of small interaction volume and large 6-dimention density expected for baryonic system produced in selected by trigger flucton-flucton interaction. To solve this problem we propose to pay attention again to the analogy between micro and macro physics effects. It is well known that such analogy (HBT-particle femtoscopy) was fruitful in the second half of XX century.

\section{Photon midrapidity spectra in $\mathrm{C}+\mathrm{Be}$ interaction.}

Photon energy spectra measured in $\mathrm{C}+\mathrm{Be}$ interactions at kinetic energy $\mathrm{T}_{0}=2.0$ and 3.2 $\mathrm{GeV} /$ nucleon for different laboratory polar angles are shown in fig. 1 and fig. 2 respectively. Slope parameters for the exponential parametrization of these spectra are presented in the upper right corner of the figures. The scale of the slope parameter $\left(\sim \mathrm{m}_{\pi}\right)$ is a typical one for cumulative process. Initial energy dependence of the slope parameter is natural for secondary particle energy range where $\mathrm{E}_{\gamma} \sim \mathrm{T}_{0}$ Angular dependence of the slope parameter in the laboratory system reflects the central mass boost.

Measured spectra go beyond the kinematical boundaries for $\mathrm{NN}$ interaction at the initial kinetic energy $\mathrm{T}_{0}=2.0$ and $3.2 \mathrm{GeV}$ respectively. Photons production in the kinematical region forbidden for the $\mathrm{NN}$ interaction can be attributed to an interaction between few nucleon short range correlations (SRC or fluctons). Kinematical boundaries for flucton-flucton interactions $\mathrm{N}_{\mathrm{i}} \mathrm{N}_{\mathrm{j}}$ are shown in fig. 3 for different $\mathrm{i}$ and $\mathrm{j}$. The upper limit of the photon energy in the measured spectra as a function of emission angle is also shown in the figure. One can see from the figure that

1. Photon spectra correspond up to 6 nucleons involved into flucton-flucton interaction.

2. Both $i>1$ and $j>1$ at least for part of measured photons because the measured spectra partly overlap $\mathrm{Be}+1$ and $1+\mathrm{C}$ kinematical boundaries.

\section{Model simulation for the baryonic system, produced in the flucton-flucton interaction.}

Phase space location for the baryonic system produced in flucton-flucton interaction has been identified in the model simulation. 
We consider flucton-flucton interactions with $\mathrm{Ni}+\mathrm{Nj}=6$ and trigger photon energy close to the kinematical limit. In this case all baryons taking part in the interaction(signal) will be produced in the opposite azimuthal direction as a compact group with relatively small internal energy( quasi binary process). To have a reasonable phase space volume for baryonic system the nucleon momentum from a signal is smeared with a parameter $\sigma_{\text {smear }}=340 \mathrm{MeV} / \mathrm{c}$ $\left(\sigma_{\mathrm{x}}=\sigma_{\mathrm{y}}=\sigma_{\mathrm{z}}=\sigma_{\text {smear }}\right)$.

Background for the flucton-flucton interaction has been estimated as follows:

1)we select UrQMD events with the number of nucleon-participants $\mathrm{N}_{\text {prod }}>5$.

2)among $\mathrm{N}_{\text {prod }}$ we find $6 \mathrm{~N}$ with minimal momentum, $\mathrm{p}_{\text {min. }}$.

3)events with $\mathrm{p}^{6 \mathrm{~N}}{ }_{\min }<\mathrm{p}_{\text {cut }}(=100 \mathrm{MeV} / \mathrm{c})$ are selected.

4)this $6 \mathrm{~N}$ are removed from each event, the remaning nucleons form the background.

Then we add this background to the $6 \mathrm{~N}$ system from flucton-flucton interaction (signal). Calculation was made for the initial energies 2,3.2, and 4GeV/nucleon. Fig.4 shows the background, the signal, and the signal+background(from left to right) nucleon distributions for $2,3.2$, and $4 \mathrm{GeV} /$ nucleon(from top to bottom) as a function of $\mathrm{y}$ and $\mathrm{p}_{\mathrm{t}}(\mathrm{x}$ and $\mathrm{y}$ axis). Signal to background(S/B) ratio in the region of the effect is shown in the table. One can see that in the region of the effect $S / B>1$ and slightly increases with initial energy. The trigger photon transverse momentum is divided between 6 nucleons in the region of the effect $p_{t}<<p_{\text {trigg. }}$ It means that in the laboratory system the emission angle of the baryonic system expected to be much smaller than for the trigger photons.

\section{Experimental setup for the study of dense and cold baryonic matter.}

The experimental set up proposed for the search and study of the baryonic system produced in the flucton-flucton interactions is shown in the fig.5. FLINT electromagnetic calorimeters $\left(\mathrm{EC}_{1}, \mathrm{EC}_{2}\right)$ will be used for the generation of trigger. Baryonic system will be identified by magnetic spectrometers(magnets $\mathrm{M}_{1}, \mathrm{M}_{2}$ in the figure, tracking, and ToF systems before and after magnets). Neutrons will be identified by thick scintillator detectors $\left(\mathrm{N}_{1}, \mathrm{~N}_{2}\right)$. Detector is proposed for the operation at ITEP external ion beam.

\section{Femtoscopy measurements for dense cold matter droplet.}

The experimental set up proposed in previous section provides the possibility of femtoscopic measurements of the dense baryonic matter droplets created in the collisions selected by the trigger. Proton-proton and proton-neutron correlations at small relative momentum will be measured. The specific features of femtoscopy in this case are a small source size and large density of fermions. Problems related to small source size have been discussed by Yu.Sinyukov in his report at the conference. Effects of large fermion density were not studied yet neither theoretically no experimentally in high energy physics. Possible analogy could be found in condensed matter physics (see, for example, [2]). 


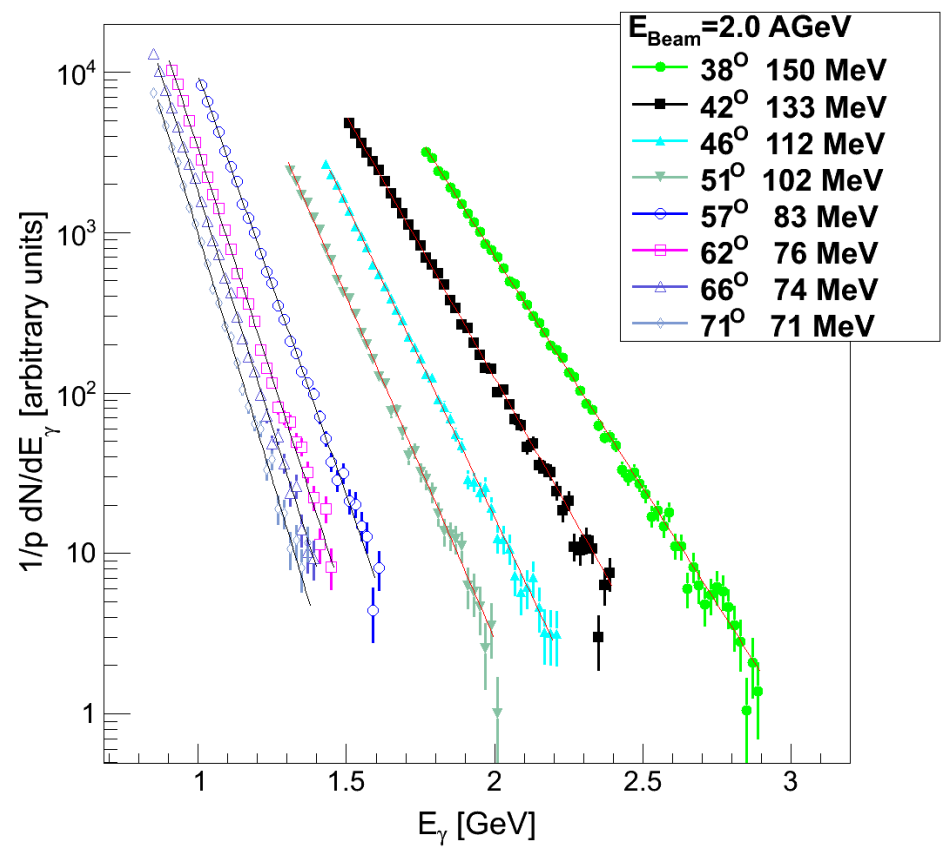

Fig.1.Photon energy spectra for initial energy $2.0 \mathrm{GeV} /$ nucleon

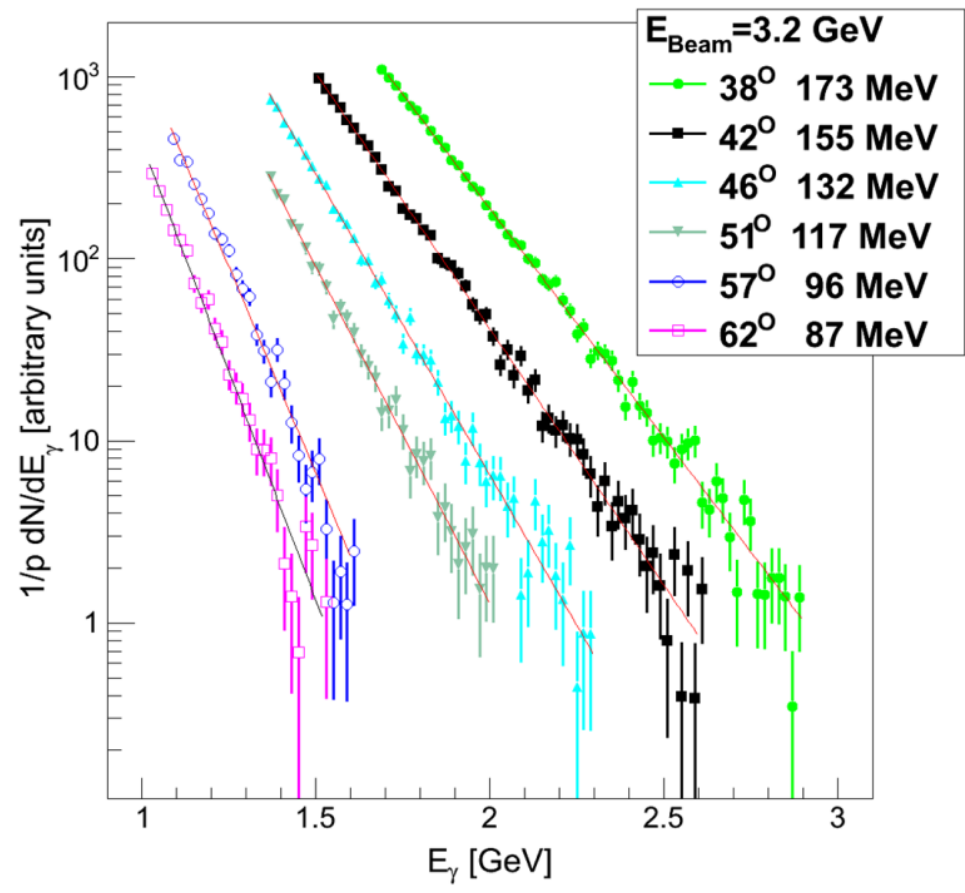

Fig.2.Photon energy spectra for initial energy $3.2 \mathrm{GeV} /$ nucleon 


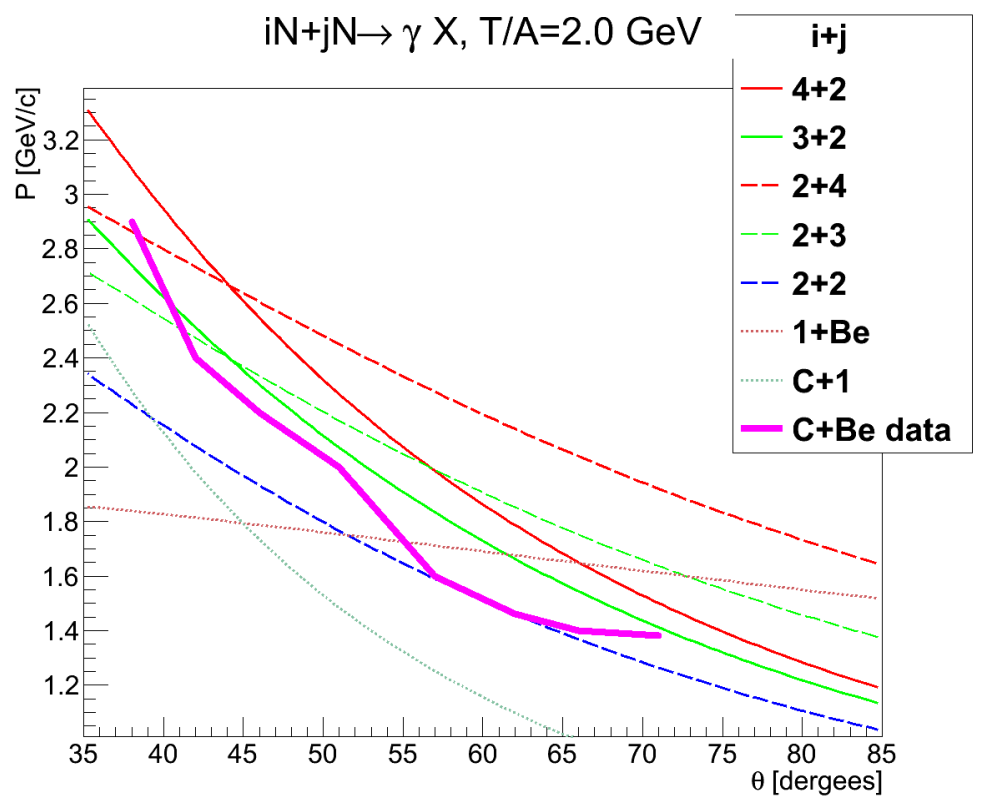

Fig.3. Kinematical boundaries for flucton-flucton interactions.

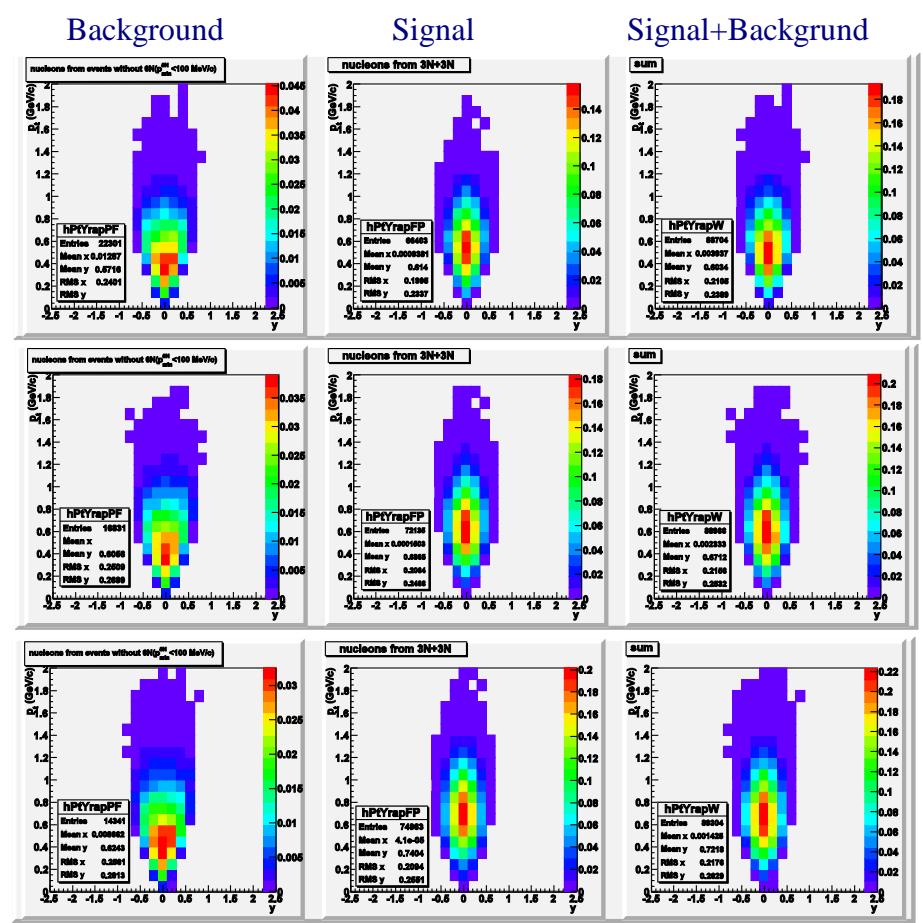

Fig.4 Background,signal, and signal+background(from left to right) nucleon distributions for $2,3.2$,and $4 \mathrm{GeV} /$ nucleon(from up to down) as a function of $\mathrm{y}$ and $\mathrm{p}_{\mathrm{t}}(\mathrm{x}$ and $\mathrm{y}$ axis) 
Table.

Signal to background ratio at the region of the effect for different initial energy

\begin{tabular}{|c|c|}
\hline T/A GeV & S/B \\
\hline 2.0 & $\sim 3$ \\
\hline 3.2 & $\sim 5$ \\
\hline 4.0 & $\sim 7$ \\
\hline
\end{tabular}

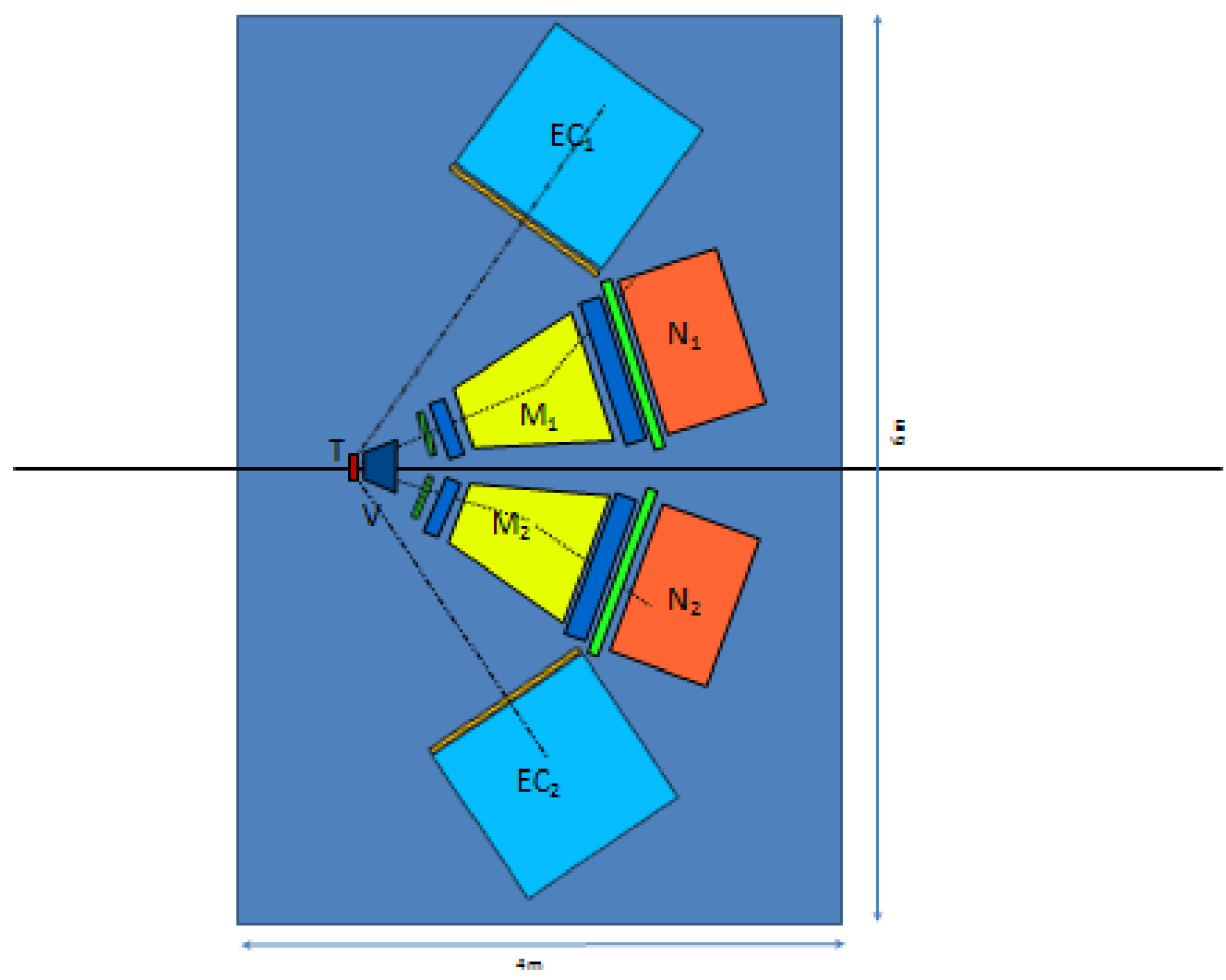

Fig.5 Experimental setup, proposed for the study of cold dense matter.

\section{References}

[1] A.Stavinskiy for the FLINT Collaboration, Proceeding of WPCF-2010, BITP,Kiev, Physics of Particle and Nuclei Letters, 8(2011)912

[2] M.Schellekens et al., Science,310, (2005)648; T.Jeltes et al., Nature, 445(2007)402 